\title{
Individualisierte Asthmatherapie in Ansätzen machbar
}

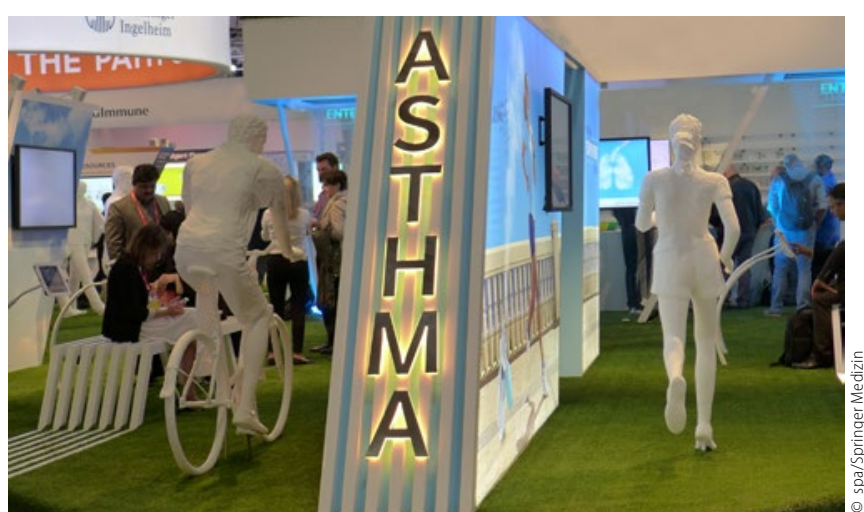

Was dem einen Patienten mit Asthma gut hilft, bringt dem anderen nicht viel. Aber es gibt im Vorfeld Hinweise, welche Therapie greift.

„Die personalisierte Asthmatherapie ist immer noch ein Traum", sagte Prof. Stanley Szefler von der Universität of Colorado/Denver. Aber es gibt erste Anhaltspunkte für eine gezielte Auswahl von Antiasthmatika, verdeutlichte er am Beispiel des Stufenplans zur

Asthmatherapie bei Kindern.

Das richtige Medikament dem richtigen Patienten zur richtigen Zeit zu geben mit dem Ziel, vollständige Asthmakontrolle zu erreichen bzw. zu erhalten. Das ist nach Angaben des Pädiaters nichts anderes als personalisierte Asthmatherapie und dafür gibt es bereits einige Auswahlkriterien.

Beispiel 1: Stufe 2 des Therapieschemas bei Kindern, die Wahl inhalativer Kortikosteroide (ICS) oder orales Montelukast. Für eine bessere Wirksamkeit von ICS wie Fluticason sprechen laut Sezfler höherer Bedarf und besseres Ansprechen auf kurz wirksame Beta-2-Mimetika (SABA), geringerer präbronchodilatatorischer $\mathrm{FEV}_{1}$-Wert und geringer $\mathrm{FEV}_{1} / \mathrm{FVC}$-Wert, geringeres Ansprechen auf den Methacholin-Provokationstest sowie höheres eosinophilisches kationisches Protein im Serum und höherer Anteil von NO in der Austatemluft (FeNO).

Beispiel 2: Stufe 3, die Frage, wann bei ungenügender Asthmakontrolle ein Step-up auf lang wirksame Betamimetika (LABA) besser ist als eine Monotherapie mit ICS. Üblich ist eine frühzeitige ICS/LABA-Kombitherapie, aber das beste Ansprechen darauf ist nur bei Kindern mit Asthma und Ekzem belegt.

Beispiel 3: Stufe 5, Intensivtherapie mit dem Anti-IgE-Antikörper Omalizumab zur Exazerbationsprophylaxe. Im Frühjahr und Herbst ist das Exazerbationsrisiko am höchsten, so Szefler, und v.a. gefährdet seien Kinder mit hohen IgE-Spiegeln, hohem FeNO, hoher Bluteosinophilie, Allergensenitivität und positiver Exazerbationsanamnese. Bei diesen Risikokindern könne durch saisonalen hoch dosierten ICS-Einsatz plus Omalizumab-Injektionen Exazerbationen am besten vorgebeugt werden.

Nachdem sich 15 Jahre lang in der Asthmatherapie bei Kindern (und auch Erwachsenen) kaum etwas verändert hat, gibt es seit kurzem Bewegung im therapeutischen Arsenal. Tiopo- tropium und ein Interleukin-5-Hemmer wurden kürzlich für asthmakranke Kinder ab 12 Jahren und Omalizumab für ab 6 Jahren zugelassen, sagte Szefler, und Studien zu Interleukin-13 und IL-4/IL-13-Hemmern bei Jugendlichen sind weit fortgeschritten. Auch für den Einsatz dieser Medikamente gibt es Biomarker bzw. Auswahlkriterien. Ein Prädiktor für das Ansprechen auf das lang wirksame Anticholinergikum ist ein gutes Ansprechen auf das SABA Arbuterol. Hohe Eosinophilenspiegel im Blut weisen auf Wirksamkeit von Interleukinblockern hin.

\section{Primärprävention}

Großer Bedarf besteht natürlich weiterhin in der Primärprävention von Asthma. Die besten Evidenzen für eine vorbeugende Wirkung gibt es für das Stillen Neugeborener über 6 Monate, sagte Prof. Anita Kozyrskyj aus Alberta. Empfehlungen zur Einnahme von Präbiotika können derzeit noch nicht gegeben werden, obwohl ein Zusammenhang zwischen dem Mikrobiom und Asthma bronchiale dokumentiert ist. Das Fehlen von Bakteroides - gefördert durch eine Sectio ebenso wie durch frühzeitigen Antibiotikaeinsatz, vermehrte Enterobakterien und Nahrungsmittelsensibilisierung korrelieren mit einem 3-fach erhöhten Asthmarisiko bei Kindern, sagte Kozyrskyj. Als Wegbereiter einer normalen Darmkolonisierung nannte sie vaginale Entbindung, keine Exposition zu Antibiotika und ausschließliches Stillen über 6 Monate.

Roland Fath

Quelle: Symposium „Deep phenotyping to improve treatment outcomes in pediatric asthma", ERS-Kongress, London

\section{Lungenentzündungen Erwachsener}

\section{Während der Schulferien stecken häufiger Pneumokokken dahinter}

Bei Lungenentzündungen Erwachsener werden während der Schulferien deutlich häufiger als in Schulzeiten Pneumokokken als Erreger identifiziert. Kontakt mit Kindern ist für Erwachsene ein Risikofaktor für Pneumokokken-Infektionen. Dies zeigt auf eindrückliche Weise die Untersuchung eines Autorenteams der Universität Nottingham. Es hatte zwischen 2008 und 2013 in zwei Krankenhäuser das Erregerspektrum von 2.221 Patienten mit ambulant erworbenen Pneumonien unter die Lupe genommen und nach Unterschieden im Erregerspektrum in den Zeiten der Schulferien gefahndet.

In 643 Fällen (29\%) lag eine Pneumokokken-Pneumonie vor. 203 dieser Pneumonien traten während der Schulferien auf. Das ist überzufällig häufig angesichts der Kürze der Schulferien. Tatsächlich war die Wahrscheinlichkeit eines Pneumokokken-Nachweises in den Schulferien nach Adjustierung für andere Variable um 35\% höher als in Schulzeiten, berichtete Studienautorin Priya Daniel. Personen mit Risikofaktoren für Pneumokokken-Infektionen wie die Großeltern können sich ganz einfach schützen, indem sie die empfohlene Pneumokokken-Schutzimpfung durchführen lassen.

Quelle: International Congress 2016 der European Respiratory Society ERS, London, 3.-7. September 2016 